\title{
GENERATION AND CHARACTERIZATION OF TWO IMMORTALIZED HUMAN OSTEOBLASTIC CELL LINES USEFUL FOR EPIGENETIC STUDIES.
}

Flor M. Pérez-Campo ${ }^{1,2}$, Tobias May³ ${ }^{3}$ Jeannette Zauers $^{3}$, Carolina Sañudo ${ }^{1}$, Jesús

Delgado-Calle ${ }^{1,4}$, Jana Arozamena ${ }^{1}$, María T. Berciano ${ }^{5}$, Miguel Lafarga ${ }^{5}$, and José A. Riancho ${ }^{1}$

1 Department of Internal Medicine, Hospital U. Marqués de Valdecilla-IDIVAL Universidad de Cantabria, 39008 Santander, Cantabria, Spain.

2 Current Address: Department of Molecular Biology, University of Cantabria, IDIVAL, Santander, Spain.

${ }^{3}$ InSCREENeX GmbH, Braunschweig, Germany.

${ }^{4}$ Current Address: Department of Anatomy and Cell Biology, Indiana University School of Medicine; Roudebush VA Medical Center, Indianapolis, Indiana, USA.

5 Department of Anatomy and Cell Biology, University of Cantabria-IDIVAL, Santander, Spain.

\section{Correspondence to:}

Jose A. Riancho, rianchoj@unican.es.

Department of Internal Medicine, Hospital U. Marqués de Valdecilla-IDIVAL Universidad de Cantabria, 39008 Santander, Cantabria, Spain.

Phone : (+34) 942201990

Fax: (+34) 942201695 


\section{ABSTRACT}

Different model systems using osteoblastic cell lines have been developed to help understand the process of bone formation. Here we report the establishment of two human osteoblastic cell lines obtained from primary cultures upon transduction of immortalizing genes. The resulting cell lines had no major differences to their parental lines in their gene expression profiles. Similar to primary osteoblastic cells, osteocalcin transcription increased following 1,25-Dihydroxyvitamin D3 treatment and the immortalized cells formed a mineralized matrix, as detected by Alizarin Red staining. Moreover, these human cell lines responded up-regulating ALPL gene expression after treatment with the demethylating agent 5-aza-2'-deoxycytidine (AzadC), as shown before for primary osteoblasts. We further demonstrate that these cell lines can differentiate in vivo, using a hidroxiapathite/tricalcium phosphate composite as a scaffold, to produce bone matrix. More importantly, we show that, these cells respond to demethylating treatment, as shown by the increase in SOST mRNA levels, the gene encoding sclerostin, upon treatment of the recipient mice with AzadC. This also confirms, in vivo, the role of DNA methylation in the regulation of SOST expression previously shown in vitro. Altogether our results show that these immortalized cell lines constitute a particularly useful model system to obtain further insight into bone homeostasis, and particularly into the epigenetic mechanisms regulating sclerostin production.

\section{KEYWORDS}

Immortalized osteoblastic cell lines, SOST, sclerostin, DNA methylation. 


\section{INTRODUCTION}

As it is the case of other primary cells, primary osteoblasts undergo a finite number of cell divisions in culture before they enter a state, known as replicative senescence [1], where they can no longer divide. Therefore, the use of primary cell lines would require a constant re-establishment of fresh cell cultures from explanted tissue, a process that can introduce significant variability in research studies[2]. In addition, primary human bone cells often show alterations of their phenotypic characteristics with increasing passage number. Other model systems, such as osteoblastic cell lines of rodent origin $[3,4]$ or human osteosarcoma cell lines [5], have been used to try to avoid this problem. However, all those systems have specific limitations. The use of cells derived from bone tumors is not advisable, as we cannot be entirely aware of the nature of the events that drove the oncogenic transformation, or whether these modifications affect their original phenotype. Also, the response capacity of these osteosarcoma cell lines to growth hormones and other factors might also be altered. Regarding the osteoblastic lines of rodent origin, their limitations come from the existence of species-specific characteristics. To overcome these problems, researches have developed human primary osteoblasic cell lines with extended replicative capacity [6,7]. In order to become useful in experimental models, theses cells not only should be able to increase the number of cell divisions that can undergo in culture, but they also require the genotypic and phenotypic characteristics of the cell line to be preserved throughout the expansion process.

We have previously shown that the expression of key genes regulating human bone homeostasis, such as SOST (encoding sclerostin), ALPL (encoding alkaline phosphatase), RANKL (encoding the receptor activator of nuclear factor 
kappa-B ligand) and OPG (encoding osteoprotegerin), is controlled by changes in the methylation status of specific CpG islands located at regulatory regions[8-10]. Despite their popularity, in our hands, osteoblastic cell lines of murine origin do not reproduce adequately the effect of demethylating agents in human systems, highlighting the importance of developing human cell lines that respond to this kind of treatments in order to allow us to study their effect in vivo.

Here we describe two new osteoblastic cell lines and compare them with already available osteoblastic cell lines and primary osteoblasts. The new cell lines maintain common markers of primary osteoblastic cells and are able to differentiate and mineralize in vitro. More importantly, these two cells lines are capable to respond to the demethylating agent AzadC not only in vitro, but also in vivo when transplanted into nude mice.

\section{MATERIALS AND METHODS}

\section{Isolation of primary osteoblasts and cell cultures}

Primary human osteoblasts were isolated from femoral heads obtained from donors undergoing hip replacement surgery due to osteoarthritis or hip fractures, as previously described [10]. In brief, small bone fragments obtained by mechanical rupture of the femoral head were washed with PBS to remove bone marrow. After the wash, bone fragments were seeded onto a T175 flask and cells were allowed to grow for 10 days in standard conditions $\left(5 \% \mathrm{CO} 2,37^{\circ} \mathrm{C}\right)$ before the first media change. When $80 \%$ confluence was reached, cells were trypsinized and used for different experiments. Culture media was Dulbecco's Modified Eagle's Medium, $10 \%$ fetal bovine serum (FBS) and 1\% penicillin/streptomycin. 
The human osteoblastic cell line MG-63 was routinely cultured in Dulbecco's Modified Eagle's Medium (DMEM, Invitrogen) supplemented with $10 \%$ FBS and antibiotics. The human osteoblastic cell line HOS-TE85 was maintained in culture with Eagle Minimum Essential Medium (MEM, Sigma-Aldrich, MO, USA) supplemented with $10 \%$ FBS and antibiotics.

For the 1,25-dihydroxy-vitamin D3 $\left(1,25(\mathrm{OH})_{2} \mathrm{D}_{3}\right)$ treatment, this factor was added to the culture media at a final concentration of $10^{-8} \mathrm{M}$ for 48 hours.

To induce osteoblastogenesis, cells were switched to osteogenic media containing $50 \mu \mathrm{M}$ ascorbic acid, $1 \mu \mathrm{M}$ dexamethasone and $10 \mathrm{mM} \beta-$ glycerophosphate for up to 4 weeks. Media was changed every $2^{\text {nd }}$ day. In order to stain calcium deposits, wells were rinsed in phosphate buffered saline (PBS), fixed in $70 \%$ ethanol for 1 hour and then rinsed with water. For the staining, $40 \mathrm{mM}$ Alizarin Red was added to the wells for 15 minutes. Excess solution was rinsed off with water and allowed to air-dry [10].

For demethylation experiments, all cells were plated at 5000 cells $/ \mathrm{cm}^{2}$ and treated for 4 days in the presence of 1 or $5 \mu \mathrm{M} 5$-Aza-2'-Deoxycytidine (AzadC) (Sigma Aldrich) prior to the analyses of gene expression.

\section{Immortalization}

For immortalization, primary cells expanded up to 3 passages were seeded on 6-well plates and infected with four different self-inactivating lentiviruses. These lentiviruses drive the expression of the following transgenes (i) SV40 large T antigen, (ii) c-myc, (iii) E7 and (iv)Id2 (sequences are available on request) from a SV40 promoter. The primary cells were infected overnight in the presence of $8 \mu \mathrm{g} / \mathrm{ml}$ polybrene. After $12 \mathrm{~h}$, the culture media containing the lentiviruses was aspirated 
and fresh cultivation media was added to the cells. Afterwards the cultivation media was renewed every two to three days for the next three weeks until colonies of proliferating cells became apparent. These proliferative colonies were pooled and further expanded.

Immortalized cell lines are available upon request.

\section{In vivo experiments}

The in vivo formation of a bone matrix was studied following the experimental model proposed by Mankani et al. [11,12]. FoxN1 ${ }^{\text {nu }}$ athymic nude mice were purchased from Harlan Laboratories and housed in a barrier facility according to animal care standards established by the European Union. All the experiments were reviewed and approved by the Animal Care Committee at the University of Cantabria.

Hidroxyapathyte/Tricalcium phosphate (HA/TCP) composites (Scaffdex) used as scaffold were purchased from Sigma. Prior to implantation in the mouse, 30-40 mgr of the pulverized composite, previously sterilized by autoclaving, were incubated with 1.5-2 million cells in $1 \mathrm{ml}$ of serum-free culture media in an eppendorf tube. Incubation was performed at $37^{\circ} \mathrm{C}$ with rotation for 90 minutes. After incubation, the tube was centrifuged briefly and excess media was removed. The composite-cell mixture was then placed subcutaneously in the back of an athymic nude mouse by using a Jamshidi-type needle of $2 \mathrm{~mm}$ internal diameter. Some mice were treated with AzadC $(700 \mathrm{mg} / \mathrm{kg}$ per week, divided in 3 doses, injected intraperitoneally).

Once removed from the mice, implants were fixed in $10 \%$ formaldehyde for 3 days at $4^{\circ} \mathrm{C}$ and decalcified in $20 \%$ EDTA in PBS (pH 7.4) for 4 weeks at $4^{\circ} \mathrm{C}$ with 
two weekly changes of the EDTA solution. After decalcification, implants were embedded in paraffin. Five microns sections were stained with hematoxylin/eosin (H\&E)[13], Masson-Goldner trichrome[14] or Sirius red[15] following standard protocols.

\section{Immunohystochemistry}

After removing paraffin and rehydration through decreasing concentration alcohol solutions and water, antigens were unmasked by exposing the slides to a $10 \mathrm{mM}$ solution of sodium citrate $\mathrm{pH} 6.0$ for 15 minutes. To quench endogenous peroxidase activity, sections were treated with $3 \%$ hydrogen peroxide in methanol for 10 minutes. After extensive washing with PBS for 20 minutes, and exposure to a blocking solution (TBST $+5 \%$ Goat serum) for 1 hour, the samples were incubated overnight with a 1/200 solution of the anti-collagen antibody (Col1a1; ab34710, Abcam, Cambridge, UK) in blocking buffer. Samples are then incubated with a secondary antibody (Biotinilated affinity purified goat anti-rabbit lgG) diluted in blocking solution for 1 hour, followed by DAB (3, 3'-diaminobenzidine) horseradish peroxidase (HRP), and counterstaining with hematoxylin.

\section{Electron microscopy}

For ultrastructural examination of the extracted implants, samples were submerged into $1 \%$ paraformaldehyde and $1 \%$ glutaraldehyde in $0.1 \mathrm{M}$ phosphate buffer, $\mathrm{pH}$ 7.4. Subsequently samples were rinsed in $0.1 \mathrm{M}$ phosphate buffer, postfixed in $2 \%$ osmium tetroxide, dehydrated in acetone and embedded in araldite (Durcupan, Fluka, Switzerland). Ultrathin sections stained with uranyl acetate and lead citrate were examined with a JEOL 201 electron microscope. 


\section{Gene expression and SOST methylation analyses.}

RNA isolation from cell cultures and removed implants and cDNA synthesis were performed as previously described[16]. Gene expression levels were measured by real time qPCR using Taqman assays (Life technologies) Expression levels were calculated relative the TATA Box Binding Protein gene (TBP) or GAPDH as $2^{-\Delta c t}$. as $2^{-\Delta c t}$. SOST promoter methylation status was determined by quantitative methylation-specific PCR (qMSP), as previously reported[8]

\section{Statistical Analysis}

Statistical significance was determined by the Student's test. Data are presented as means \pm standard error of the mean values.

\section{RESULTS}

MORPHOLOGICAL AND TRANSCRIPTIONAL CHARACTERIZATION OF THE IMMORTALIZED CELL LINES.

The primary osteoblastic cultures were isolated from the femoral head of an osteoarthritic patient subjected to hip replacement and a patient that suffered an osteoporotic hip fracture. The two immortalized cell lines derived from osteoblasts obtained from these two patients were named OB50Li and OB52Li, respectively.

After isolation and expansion of the primary cells, they were transduced with four different lentiviral vectors encoding for (i) SV40 large T antigen (TAg), (ii) cmyc, (iii) E7 and (iv) inhibitor of DNA binding 2 (Id2), a dominant negative helix- 
loop-helix protein. These genes were identified in a previous screen as factors that facilitate the immortalization of primary human osteoblasts (Lipps et al., In preparation). For selection of transduced primary cells, we utilized the difference in proliferation capacity between the primary human osteoblasts (low proliferation capacity) and the infected cells (high proliferation capacity) and maintained the infected cells for three weeks with regular media exchange until colonies of proliferating cells arose. The individual colonies of immortalized cells were then pooled and further expanded. The resulting polyclonal cell lines showed robust proliferation and were truly immortalized, as they could be cultivated for more than hundred cumulative population doublings showing no signs of senescence.

The cell morphology of the OB50Li and OB52Li cell lines under phasecontrast microscopy was clearly different from that of the original primary cell lines. While the primary cells showed the typical spindly shape appearance, the immortalized cell lines showed a polygonal shape (Figure 1A). The immortalized cell lines typically grew from the initial cell colonies with in a sheet-like pattern until confluence was reached.

To assay which conditions promote a more sustained growth of these cells lines, immortalized cells were seeded in cultures containing different percentages of FBS. The proliferation of the two cell lines was enhanced in media with increasing concentrations of serum between 1 and 10\% (Figure 1B). Both lines proliferated stably until they reached confluence, although the OB52Li cell line seemed to be slightly less proliferative with a higher doubling time (12.5 hours for OB50Li versus 14.5 hours for OB52Li).

The osteogenic nature of the cell lines can be characterized in vitro by assessing the expression of temporally regulated osteogenic markers. Mature 
osteoblasts produce bone matrix constituents such as osteocalcin (encoded by BGLAP) [17]. Osteoblasts also regulate bone matrix mineralization[18] through the production of large amounts of alkaline phosphatase, encoded by the ALPL gene [19]. Expression analysis of the genes encoding these two proteins revealed that the two immortalized cell lines expressed both osteogenic markers, although to a different extent (Figure 1C). BGLAP expression levels were similar in the immortalized cell lines and in non-transformed primary human osteoblasts (hOBs). In fact, the expression levels of the two immortalized cell lines seem to be more similar to those of the primary osteoblasts that those of the MG-63 cell line. Regarding ALPL, the expression level of this gene in primary osteoblasts was considerably higher than that on the osteoblastic cell line MG63, as we have previously described [10] (Figure 1C). ALPL expression level in the immortalized cell lines was intermediate between primary osteoblasts and MG-63. The expression of type 1 collagen (COL1A1) and other genes typical of the osteoblastic lineage was lower in the immortalized cell lines than in primary osteoblasts (figure 1D).

\section{IN VITRO RESPONSE TO THE DEMETHYLATING AGENT 5-AZA-2'- DEOXYCITIDINE AND TO VITAMIN D}

In line with previous reports using primary osteoblasts [10], we observed that ALPL expression by the OB50Li and OB52Li cell lines increased significantly in response to AzadC ( $p<0.05$ versus baseline for all cell types), thus showing that the response to this inhibitor of DNA methyltransferases is indeed preserved in the OB50Li and OB52Li cell lines (Figure 2A). 
The vitamin $D$ active metabolite $1,25(\mathrm{OH})_{2} \mathrm{D}_{3}$ is one of the main endocrine factors regulating bone homeostasis. It can regulate the activity of both bone forming cells (osteoblasts) and bone resorbing cells (osteoclasts)[20]. $1,25(\mathrm{OH})_{2} \mathrm{D}_{3}$ is known to control osteoblast proliferation, differentiation and mineralization[21] by inducing the production of alkaline phosphatase [22-26] and osteocalcin $[27,28]$.

We cultured the immortalized osteoblastic cell lines in the presence and absence of $1,25(\mathrm{OH})_{2} \mathrm{D}_{3}$ and analysed the expression of $\mathrm{BGLAP}$, the gene encoding osteocalcin, which is involved in the mineralization process. (Figure 2B). Quantitative real time PCR (qPCR) analysis demonstrated, in both cell lines, an upregulation of the osteoblastic marker BGLAP in response to $1,25(\mathrm{OH})_{2} \mathrm{D}_{3}$, which is consistent with previous results obtained in primary osteoblasts[29].

\section{MINERALIZATION}

In order to check whether these immortalized cell lines were able to produce an osteoid-like mineralized matrix, we incubated confluent hOBs and immortalized osteoblasts for 28 days in an osteogenic culture medium and determined their mineralization potential by Alizarin red staining at different time points (Figure 2C). Figure 2C clearly shows that both immortalized cell lines are able to form a mineralized matrix in response to stimuli inducing osteogenic differentiation. ALPL is mainly expressed during the initiation of the mineralization process and is soon observed on the cell surface, however its expression declines later in the developmental program. Consistent with this, we found a decrease in the expression levels of ALPL during the mineralization process (Figure 2D), which is also consistent with previous reports in primary osteoblasts [10]. 
The final stage of osteoblast differentiation is also accompanied by the upregulation of genes more characteristic of the osteocyte phenotype, such as SOST, encoding sclerostin, or the gene encoding the fibroblast growth factor 23 (FGF23). Similar to the results previously obtained with primary osteoblasts, none of these transcripts were detectable by qPCR in either the non-transformed or the immortalized osteoblasts (data not shown).

\section{ANALYSIS OF THE BONE FORMING ACTIVITY OF THE IMMORTALIZED OSTEOBLASTIC CELL LINES IN VIVO.}

In order to assay the bone forming capacity of the OB50Li and OB52Li lines in vivo, both cell lines were seeded onto an HA/TCP scaffold prior to subcutaneous implantation in FoxN1 ${ }^{\text {nu }}$ athymic nude mice. As control, we also performed the same procedure with human primary osteoblasts (hOBs) and the osteosarcoma cell line HOS-TE85. After three months, the implants were surgically removed and the extent of new bone formation was analysed by gene expression and histological techniques. It is important to note that no tumor formation was observed in the nude mice after the three months period demonstrating that the novel cell lines are immortalized but not oncogenically transformed.

The H\&E staining revealed the existence of cell infiltrations between the scaffold particles. The scaffold pores contained a large number of cells distributed throughout the scaffold and/or bone matrix, irrespective of the cell type initially seeded in the scaffold (Figure 3A, H\&E column). Immunohistochemistry analysis

also showed that all implants were immuno-reactive for Collagen type I (Figure 3A, IHQ COL1a1 column) with a very low background labelling, although its presence 
was more noticeable in the case of the primary osteoblasts and the immortalized cell lines OB50Li and OB52Li (Figure 3A, IHQ COL1a1 column). This was also shown using the Masson-Goldner technique (Figure 3A, Masson column), a widely used histological technique that stains collagen in a blue/green colour. Bone-type matrix was not very abundant and looked somewhat immature, but immortalized cells and primary non-transformed cells laid similar amounts of matrix. Interestingly, observation of the implants at higher magnification, revealed some cells embedded within the matrix and surrounded by a clear region, resembling the morphology of osteocytes within osteocytic lacunae (Figure 3B).

The samples were stained with Sirius Red to observe the disposition of collagen fibres under polarized light. In Figure 4A it is possible to observe clear bright areas that would correspond to the birefringence of collagen fibres structured in an antiparallel disposition, characteristic of the organized lamellar bone. Additionally, the auto-fluorescence of bone was also detected in these samples when observed under green fluorescent light (Figure 4B).

Electron microscopy studies confirmed the presence of collagen fibres laid in an antiparallel orientation (Figure 4C1), dense mineralization vesicles (Figure 4C2), cells embedded in the collagen matrix (Figure 4C3) and polarized osteoblasts with extensive rough endoplasmic reticulum (Figure 4C4).

To analyse the in vivo osteogenic signature of the immortalized cells, we isolated mRNA from the implants and carried out quantitative PCR analysis for the osteogenic markers ALPL, COL1A1 and SOST using oligonucleotides specific for the detection of the human genes. ALPL and SOST were expressed in implants of both immortalized cell lines, although to a different extent, as shown in Figure $5 \mathrm{~A}$. 
Also, confirming the histological results, the expression of COL1A1 was detected in the implants.

\section{IN VIVO RESPONSE TO THE DEMETHYLATING AGENT AZA-DEOXYCITIDINE}

In our hands, osteoblastic cell lines of murine origin do not reproduce adequately the effect of demethylating agents in human systems. We tested whether the immortalized cell lines were able to respond to treatment with the demethylating agent AzadC in vivo. For this purpose, mice harbouring implants were treated with AzadC for a month. Control mice were injected with an equivalent volume of saline solution (Figure 5B). As shown in Figure $5 \mathrm{C}$, treatment with AzadC significantly increased the expression levels of sclerostin in both immortalized cell lines and in the human osteosarcoma cell line HOS-TE85 to a similar level. In line with this, by using qMSP we confirmed that the methylation of the promoter of the SOST gene was decerased by AzadC treatment (not shown).

\section{DISCUSSION}

Osteoporosis is a disorder characterized by a low bone mass. It results from the uncoupled activity of bone resorbing osteoclasts and bone-forming osteoblasts. Given the high prevalence of osteoporosis among postmenopausal women and old men, intense research is on going in order to get a deeper understanding of the disease pathogenesis and to discover new drug targets. In fact, although potent anti-resorbing drugs are available, the armamentarium to stimulate bone formation

is much more limited. Hence there is a strong interest in the development of 
experimental models allowing to effectively screen interventions promoting bone formation.

Osteoblasts are responsible for the formation of new bone and therefore a tight control of their activity is key for skeletal homeostasis. A variety of cell lines from mouse and human origins have been described and are frequently used, along with primary osteoblasts, as models to study osteoblast activity. However, given the rapid loss of replicative potential of the primary human osteoblasts, there is a need for alternative models. On the other hand, spontaneously immortalized human osteoblastic cell lines may accumulate a number of DNA changes and they may not preserve adequately the normal phenotype. In this work, we describe the generation and characterization of two new human osteoblastic cell lines that were immortalized by the controlled transfection of a set of survival genes. They had a number of characteristics resembling those of normal osteoblasts, including the expression of genes typical of osteoblasts and the ability to form an osteoid-like matrix, both in vitro and in vivo.

We showed that these cells appeared to preserve many of the characteristics of primary osteoblasts. They are able to proliferate normally and express typical osteogenic markers, such as BGLAP and ALPL. Indeed BGLAP expression levels in the OB50Li and OB52Li cell lines are closer to the primary cell lines that those of MG-63 cells, a popular osteoblastic line. Small differences in the osteocalcin transcriptional levels between the two immortalized cell lines could be related to difference in age of the donors or in their bone health status as previously described $[30,31]$. We detected a lower level of expression of ALPL in the immortalized cell lines compared to primary human osteoblasts, a difference that could indicate a more advanced maturation stage. Since the levels of ALPL have 
been shown to be regulated by the methylation levels of its promoter region, this lower expression level of ALPL in the immortalized cell lines probably reflects a higher level of methylation of the ALPL promoter in those lines. In line with this hypothesis, we have shown that the immortalized cell lines are able to respond, in vitro, to the demethylating agent AzadC. In contrast, primary osteoblasts have an already hypomethylated ALPL promoter and, consequently, do not increase gene expression in response to AzadC [10]. Likewise, the immortalized cell lines are able to respond to $1,25(\mathrm{OH})_{2} \mathrm{D}_{3}$ by up-regulating $\mathrm{BGLAP}$, contrasting with the absence of response seen in MG-63 cells, indicating that the regulatory mechanisms driven by vitamin $D$ are also preserved in these cell lines. The small differences between the two immortalized cell lines might again reflect different stages of maturation [32,33]. Moreover, when maintained in an osteogenic culture medium the two immortalized cell lines are able to form a mineralized matrix. This is concomitant with the downregulation of ALPL, a marker which expression is known to decrease rapidly during the mineralization process, as shown in primary osteoblast cultures. However, we were not able to detect the expression of osteocyte specific genes, such as SOST or FGF23, in cultures of the immortalized cells lines maintained for several weeks in vitro. Similarly, those genes were not detected in long-term cultures of primary osteoblasts. These results suggest that current in vitro models are not able to induce a terminal differentiation of osteoblasts into osteocytes.

Notably, the two cell lines are able to produce bone matrix in vivo, when cells are seeded in a HA/TCP scaffold and implanted in extra-skeletal tissues of athymic nude mice. For bone tissue to develop from cells implanted in vivo, a scaffold is needed to provide both a surface and a space for the growth of new cells. Hydroxyapatite/tricalcium phosphate (HA/TCP) particles mimic the natural bone 
matrix in humans and have been shown to provide an appropriate environment for the growth of bone tissue [34-36]. The analysis by qPCR confirmed the expression of different bone markers in the implants seeded with the immortalized cells lines. These results confirm that the HA/TCP scaffolds can support the growth of the immortalized cell lines, and that these cell lines can differentiate to produce human bone matrix in vivo.

Recent findings have shown that DNA methylation of regulatory regions is a key mechanism controlling expression of osteogenic genes. Of particular interest is the regulation of SOST, the gene encoding sclerostin, an important inhibitor of bone formation. However, in our hands, mouse immortalized osteoblastic cell lines do not seem to respond in vivo to the demethylating agent AzadC and therefore are not a good model to study the regulation of osteoblast differentiation by DNA methylation in general, and SOST/sclerostin regulation in particular. Importantly, we have shown that the two immortalized cell lines presented here are able to respond in vivo to the treatment with AzadC by up-regulating SOST expression. Sclerostin is a critical factor in the regulation of bone formation. In fact, neutralizing anti-sclerostin antibodies are being developed as a new therapy for osteoporosis $[37,38]$. However, the mechanisms regulating sclerostin expression are not well established. This is largely due to the fact that sclerostin production is limited to osteocytes and to the absence of human osteocytic cell lines. The fact that our immortalized cell lines respond to AzadC treatment by up-regulating the expression of the SOST gene after transplantation into nude mice confirms previous results in vitro and makes these cell lines useful models to study the regulatory effects of DNA methylation on cells of the osteoblastic lineage in vivo. 


\section{AKNOWLEDGEMENTS}

This research was supported by a grant from the Spanish Ministry of Health and the

"Instituto de Salud Carlos III" (P112-0615). C. Sanudo is funded by a grant from the IDIVAL (Instituto de Investigacion Marques de Valdecilla).

\section{CONFLICT OF INTEREST.}

The authors declare no conflict of interest.

\section{REFERENCES}

1. Stewart SA, Weinberg RA (2002) Senescence: does it all happen at the ends? Oncogene 21:627-630.

2. Lipps C, May T, Hauser H, Wirth D (2013) Eternity and functionality - rational access to physiologically relevant cell lines. Biol Chem 394:1637-1648.

3. Sudo H, Kodama HA, Amagai Y, Yamamoto S, Kasai S (1983) In vitro differentiation and calcification in a new clonal osteogenic cell line derived from newborn mouse calvaria. J Cell Biol 96:191-198.

4. Benayahu D, Kletter Y, Zipori D, Wientroub S (1989) Bone marrow-derived stromal cell line expressing osteoblastic phenotype in vitro and osteogenic capacity in vivo. J Cell Physiol 140:1-7.

5. Rodan SB, Imai Y, Thiede MA, Wesolowski G, Thompson D, Bar-Shavit Z, Shull S, Mann K, Rodan GA (1987) Characterization of a human osteosarcoma cell line (Saos-2) with osteoblastic properties. Cancer Res 47:4961-4966.

6. Bodine PV, Trailsmith M, Komm BS (1996) Development and characterization of a conditionally transformed adult human osteoblastic cell line. J Bone Miner Res 11:806-819.

7. Harris SA, Enger RJ, Riggs BL, Spelsberg TC (1995) Development and characterization of a conditionally immortalized human fetal osteoblastic cell line. J Bone Miner Res 10:178-186.

8. Delgado-Calle J, Sanudo C, Bolado A, Fernandez AF, Arozamena J, Pascual-Carra MA, Rodriguez-Rey JC, Fraga MF, Bonewald L, Riancho JA (2012) DNA methylation contributes to the regulation of sclerostin expression in human osteocytes. J Bone Miner Res 27:926-937.

9. Delgado-Calle J, Sanudo C, Fernandez AF, Garcia-Renedo R, Fraga MF, Riancho JA (2012) Role of DNA methylation in the regulation of the RANKLOPG system in human bone. Epigenetics 7:83-91.

10. Delgado-Calle J, Sanudo C, Sanchez-Verde L, Garcia-Renedo RJ, Arozamena J, Riancho JA (2011) Epigenetic regulation of alkaline phosphatase in human cells of the osteoblastic lineage. Bone 49:830-838. 
11. Mankani MH, Kuznetsov SA, Marshall GW, Robey PG (2008) Creation of new bone by the percutaneous injection of human bone marrow stromal cell and HA/TCP suspensions. Tissue Eng Part A 14:1949-1958.

12. Mankani MH, Kuznetsov SA, Avila NA, Kingman A, Robey PG (2004) Bone formation in transplants of human bone marrow stromal cells and hydroxyapatite-tricalcium phosphate: prediction with quantitative CT in mice. Radiology 230:369-376.

13. Cowan CM, Shi YY, Aalami OO, Chou YF, Mari C, Thomas R, Quarto N, Contag CH, Wu B, Longaker MT (2004) Adipose-derived adult stromal cells heal critical-size mouse calvarial defects. Nat Biotechnol 22:560-567.

14. Rentsch C, Schneiders W, Manthey S, Rentsch B, Rammelt S (2014) Comprehensive histological evaluation of bone implants. Biomatter 4.

15. Tullberg-Reinert $H$, Jundt $G$ (1999) In situ measurement of collagen synthesis by human bone cells with a sirius red-based colorimetric microassay: effects of transforming growth factor beta2 and ascorbic acid 2phosphate. Histochem Cell Biol 112:271-276.

16. Delgado-Calle J, Arozamena J, Perez-Lopez J, Bolado-Carrancio A, Sanudo C, Agudo G, de la Vega R, Alonso MA, Rodriguez-Rey JC, Riancho JA (2013) Role of BMPs in the regulation of sclerostin as revealed by an epigenetic modifier of human bone cells. Mol Cell Endocrinol 369:27-34.

17. Lee NK, Sowa H, Hinoi E, Ferron M, Ahn JD, Confavreux C, Dacquin R, Mee PJ, McKee MD, Jung DY, Zhang Z, Kim JK, Mauvais-Jarvis F, Ducy P, Karsenty G (2007) Endocrine regulation of energy metabolism by the skeleton. Cell 130:456-469.

18. Boskey AL (1998) Biomineralization: conflicts, challenges, and opportunities. J Cell Biochem Suppl 30-31:83-91.

19. Whyte MP (1994) Hypophosphatasia and the role of alkaline phosphatase in skeletal mineralization. Endocr Rev 15:439-461.

20. Jones G, Hogan DB, Yendt E, Hanley DA (1996) Prevention and management of osteoporosis: consensus statements from the Scientific Advisory Board of the Osteoporosis Society of Canada. 8. Vitamin D metabolites and analogs in the treatment of osteoporosis. CMAJ 155:955961.

21. van Leeuwen JP, van Driel M, van den Bemd GJ, Pols HA (2001) Vitamin D control of osteoblast function and bone extracellular matrix mineralization. Crit Rev Eukaryot Gene Expr 11:199-226.

22. Wong MM, Rao LG, Ly H, Hamilton L, Tong J, Sturtridge W, McBroom R, Aubin JE, Murray TM (1990) Long-term effects of physiologic concentrations of dexamethasone on human bone-derived cells. J Bone Miner Res 5:803813.

23. Beresford JN, Gallagher JA, Russell RG (1986) 1,25-Dihydroxyvitamin D3 and human bone-derived cells in vitro: effects on alkaline phosphatase, type I collagen and proliferation. Endocrinology 119:1776-1785.

24. Shakoori AR, van Wijnen AJ, Bortell R, Owen TA, Stein JL, Lian JB, Stein GS (1994) Variations in vitamin D receptor transcription factor complexes associated with the osteocalcin gene vitamin $D$ responsive element in osteoblasts and osteosarcoma cells. J Cell Biochem 55:218-229.

25. Rao LG, Wylie JN, Kung Sutherland MS, Murray TM (1996) 17 betaoestradiol enhances the stimulatory effect of 1,25-dihydroxyvitamin D3 on 
alkaline phosphatase activity in human osteosarcoma SaOS-2 cells in a differentiation-dependent manner. J Endocrinol 148:181-187.

26. Sutherland MK, Hui DU, Rao LG, Wylie JN, Murray TM (1996) Immunohistochemical localization of the estrogen receptor in human osteoblastic SaOS-2 cells: association of receptor levels with alkaline phosphatase activity. Bone 18:361-369.

27. Chen TL, Mallory JB, Hintz RL (1991) Dexamethasone and 1,25(OH)2 vitamin D3 modulate the synthesis of insulin-like growth factor-I in osteoblast-like cells. Calcif Tissue Int 48:278-282.

28. Scharla SH, Strong DD, Mohan S, Baylink DJ, Linkhart TA (1991) 1,25Dihydroxyvitamin D3 differentially regulates the production of insulin-like growth factor I (IGF-I) and IGF-binding protein-4 in mouse osteoblasts. Endocrinology 129:3139-3146.

29. Maruotti N, Corrado A, Grano M, Colucci S, Cantatore FP (2009) Normal and osteoporotic human osteoblast behaviour after 1,25-dihydroxy-vitamin $\mathrm{D}(3)$ stimulation. Rheumatol Int 29:667-672.

30. Vanderschueren D, Gevers G, Raymaekers G, Devos P, Dequeker J (1990) Sex- and age-related changes in bone and serum osteocalcin. Calcif Tissue Int 46:179-182.

31. Westacott Cl, Webb GR, Warnock MG, Sims JV, Elson CJ (1997) Alteration of cartilage metabolism by cells from osteoarthritic bone. Arthritis Rheum 40:1282-1291.

32. Matsumoto $\mathrm{T}$, Igarashi $\mathrm{C}$, Takeuchi $\mathrm{Y}$, Harada S, Kikuchi T, Yamato H, Ogata E (1991) Stimulation by 1,25-dihydroxyvitamin D3 of in vitro mineralization induced by osteoblast-like MC3T3-E1 cells. Bone 12:27-32.

33. Ishida H, Bellows CG, Aubin JE, Heersche JN (1993) Characterization of the 1,25- $(\mathrm{OH}) 2 \mathrm{D} 3$-induced inhibition of bone nodule formation in long-term cultures of fetal rat calvaria cells. Endocrinology 132:61-66.

34. Shaw TJ, Senterman MK, Dawson K, Crane CA, Vanderhyden BC (2004) Characterization of intraperitoneal, orthotopic, and metastatic xenograft models of human ovarian cancer. Mol Ther 10:1032-1042.

35. Suzuki Y, Yamashita R, Shirota M, Sakakibara Y, Chiba J, MizushimaSugano J, Nakai K, Sugano S (2004) Sequence comparison of human and mouse genes reveals a homologous block structure in the promoter regions. Genome Res 14:1711-1718.

36. Valentiner U, Haane C, Nehmann N, Schumacher U (2009) Effects of bortezomib on human neuroblastoma cells in vitro and in a metastatic xenograft model. Anticancer Res 29:1219-1225.

37. Eddleston A, Marenzana M, Moore AR, Stephens P, Muzylak M, Marshall D, Robinson MK (2009) A short treatment with an antibody to sclerostin can inhibit bone loss in an ongoing model of colitis. J Bone Miner Res 24(10):1662-1671.

38. Li X, Ominsky MS, Warmington KS, Morony S, Gong J, Cao J, Gao Y, Shalhoub V, Tipton B, Haldankar R, Chen Q, Winters A, Boone T, Geng Z, Niu QT, Ke HZ, Kostenuik PJ, Simonet WS, Lacey DL, Paszty C (2009) Sclerostin antibody treatment increases bone formation, bone mass, and bone strength in a rat model of postmenopausal osteoporosis. J Bone Miner Res 24:578-588. 


\section{FIGURE LEGENDS}

FIGURE 1. Phenotypic characteristics of the immortalized cell lines. (A) Morphology of cells from primary osteoblastic cultures and their immortalized counterparts. (B) Growth curve of the OB50Li and OB53Li cell lines growing in media supplemented with increasing concentrations of Fetal Bovine Serum. (C) Comparison of osteocalcin (BGLAP) and alkaline phosphatase (ALPL) expression levels between the human sarcoma cell line MG-63, primary human osteoblasts (OBs) and the two immortalized cell lines. (D) Expression levels of other genes in OBs and the cell lines. Values reflect averages of triplicate samples. Bars represent standard error of the mean.

FIGURE 2. Response to AzadC and vitamin D and mineralization in vitro. (A) ALPL expression in OBs and the immortalized cells in response to AzadC ( $5 \mu \mathrm{M}$ for 3 days). Bars represent the mean fold change in comparison with baseline expression in a representative triplicate experiment. (B) Quantitative PCR showing the relative expression levels of BGLAP in response to Vitamin D treatment. The graph represents the average values from 3 independent cultures. Bars represent standard error of the mean values. (C) Left panel. Time course of calcification in human primary osteoblasts (hOBs) and in the immortalized cell lines. Picture shows Alizarin Red staining of the mineralized matrix. Right panel. qPCR of ALPL expression during the mineralization process in the immortalized cell lines. The graph represents the average values from 3 independent cultures. Bars represent standard error of the mean values. 
FIGURE 3. H\&E and Masson-Goldner staining, and Col1a1 immunohistochemistry indicating bone forming activity of the immortalized osteoblastic cell lines in vivo. (A) Pictures show histological analysis of sections obtained from decalcified implants. H\&E (a, d, g and j) and Masson-Goldner staining (c, f, i and I) showing mineralized matrix. Immunohistochemistry analysis of the correspondent sections using a Col1a1 antibody (b, e, h and k) where arrows indicate regions rich in COL1a1. Magnification 8X. (B) Arrows indicate osteocyteslike cells surrounded by lacunae and immersed in the mineralized matrix. Asterisks mark some of the HA/TCP scaffold particles. Magnification 40X.

FIGURE 4. Sirius Red staining and fluorescence and electron microscopy analysis of histological sections. (A) Polarization microscopy of Sirius red stained collagen fibres, showing birefringence of collagen enriched areas. Scale bar $5 \mu \mathrm{m}$. (B) H \& E stained slides observed under green fluorescent show the typical auto-fluorescence of collagen fibers. Scale bar $5 \mu \mathrm{m}$. (C) Electron microscopy analysis of the implants seeded with the OB52Li immortalized cell lines showing collagen fibres laid in an antiparallel orientation (1), dense mineralization vesicles (2), cells embedded in the collagen matrix (3) and polarized osteoblasts with an active reticulum (4). Scale bars: $1,2 \mu \mathrm{m} ; 2-4,5 \mu \mathrm{m}$.

FIGURE 5. In vivo expression of osteogenic markers and response to AzadC response. (A) Graphs show the in vivo expression of different osteogenic markers (SOST, ALPL and COL1A1) in the implants initially seeded with the immortalized cell lines (OB50Li and OB52Li), with human primary osteoblasts (Obs) and with the 
human osteosarcoma cell line HOS-TE85 (HOS). Primers used were specific for the amplification of the human transcripts. (B) 2 months after implantation mice harbouring the implants were treated with AzadC for four weeks before implant removal. Control mice were injected with saline solution. (C) Graphs represent in vivo expression levels of sclerostin in the control mice and mice treated with AzadC for four weeks. The graph represents the average values from 3 independent cultures. Bars represent standard error of the mean values. $P<0.05\left({ }^{*}\right), P<0.01\left(^{* *}\right)$. 
A
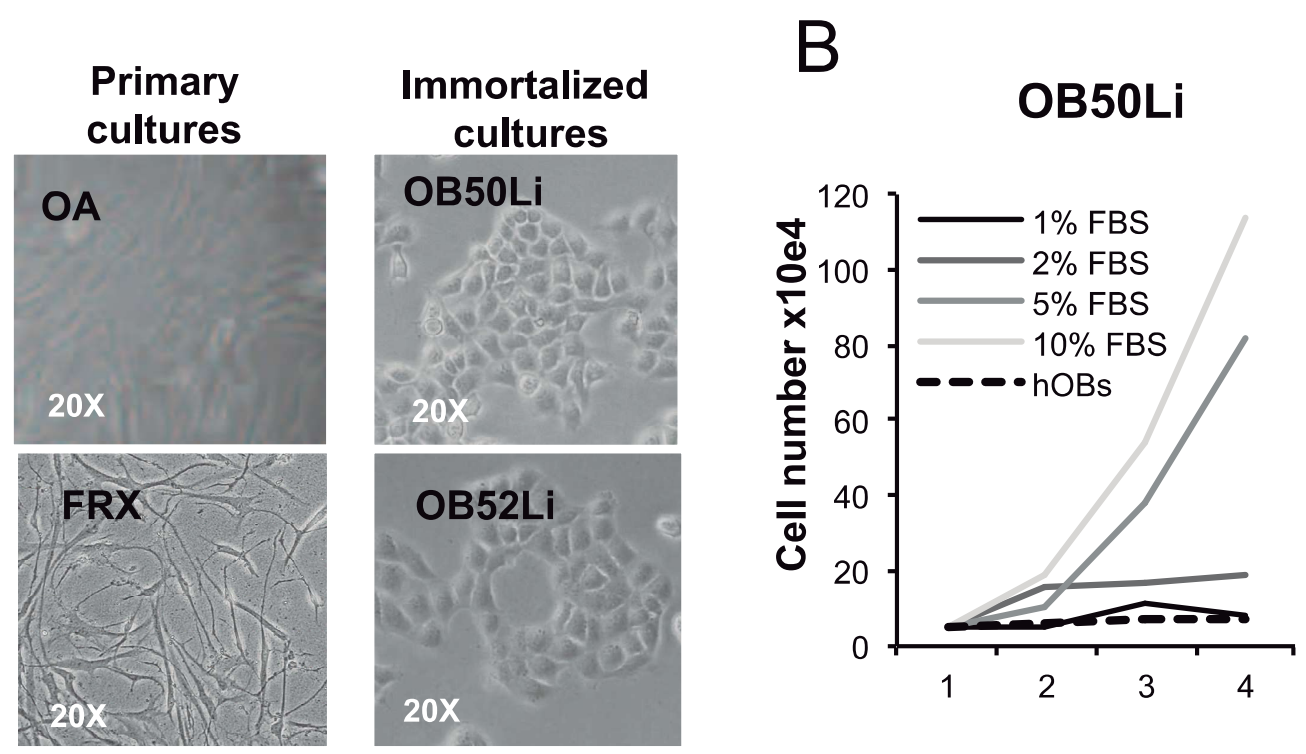

OB52Li

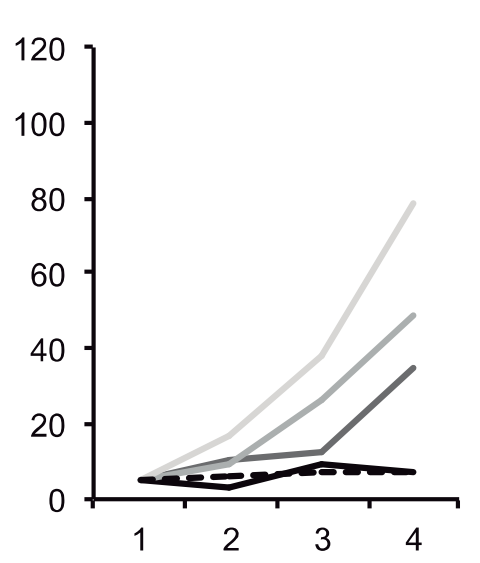

C

D
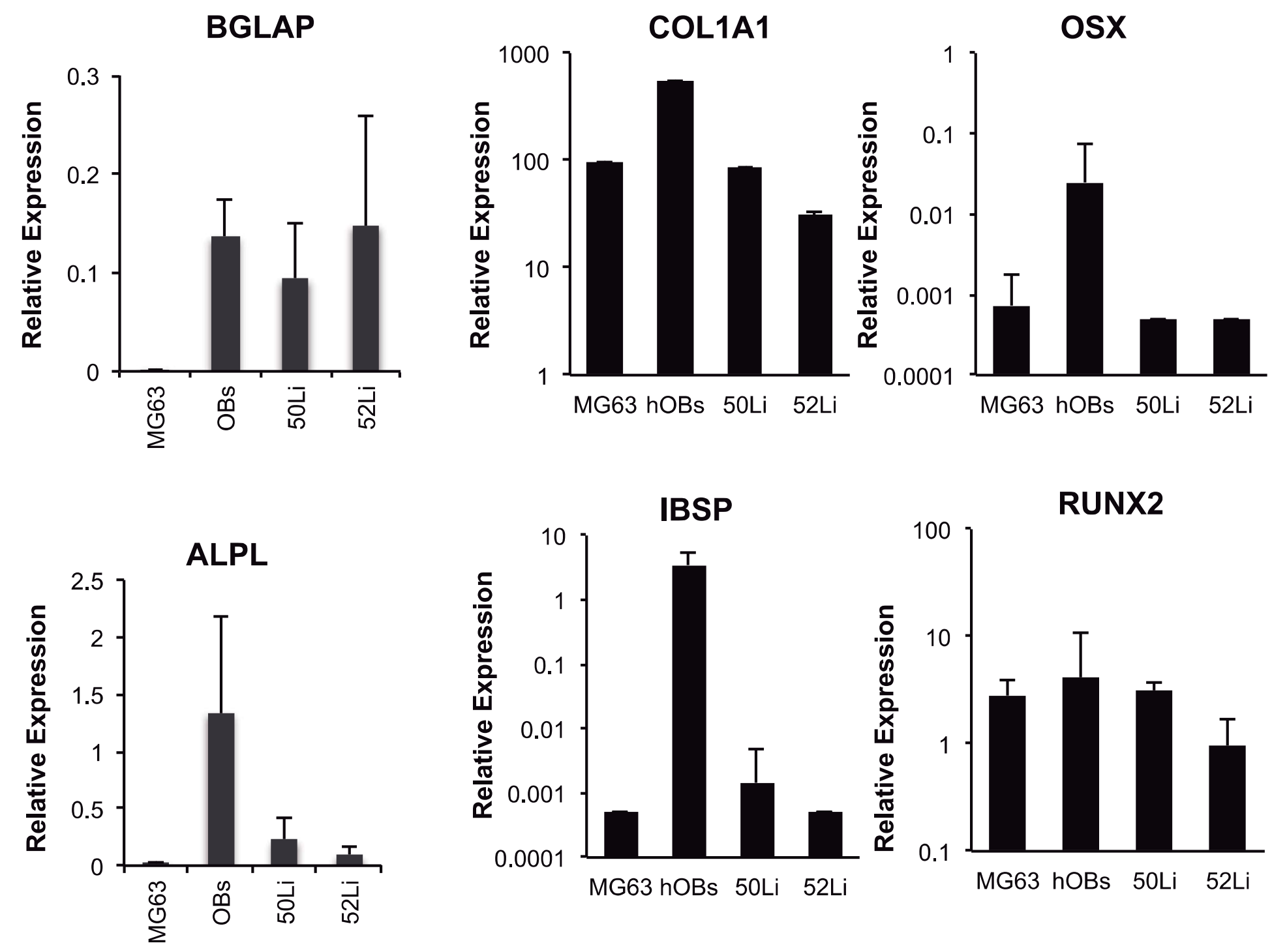

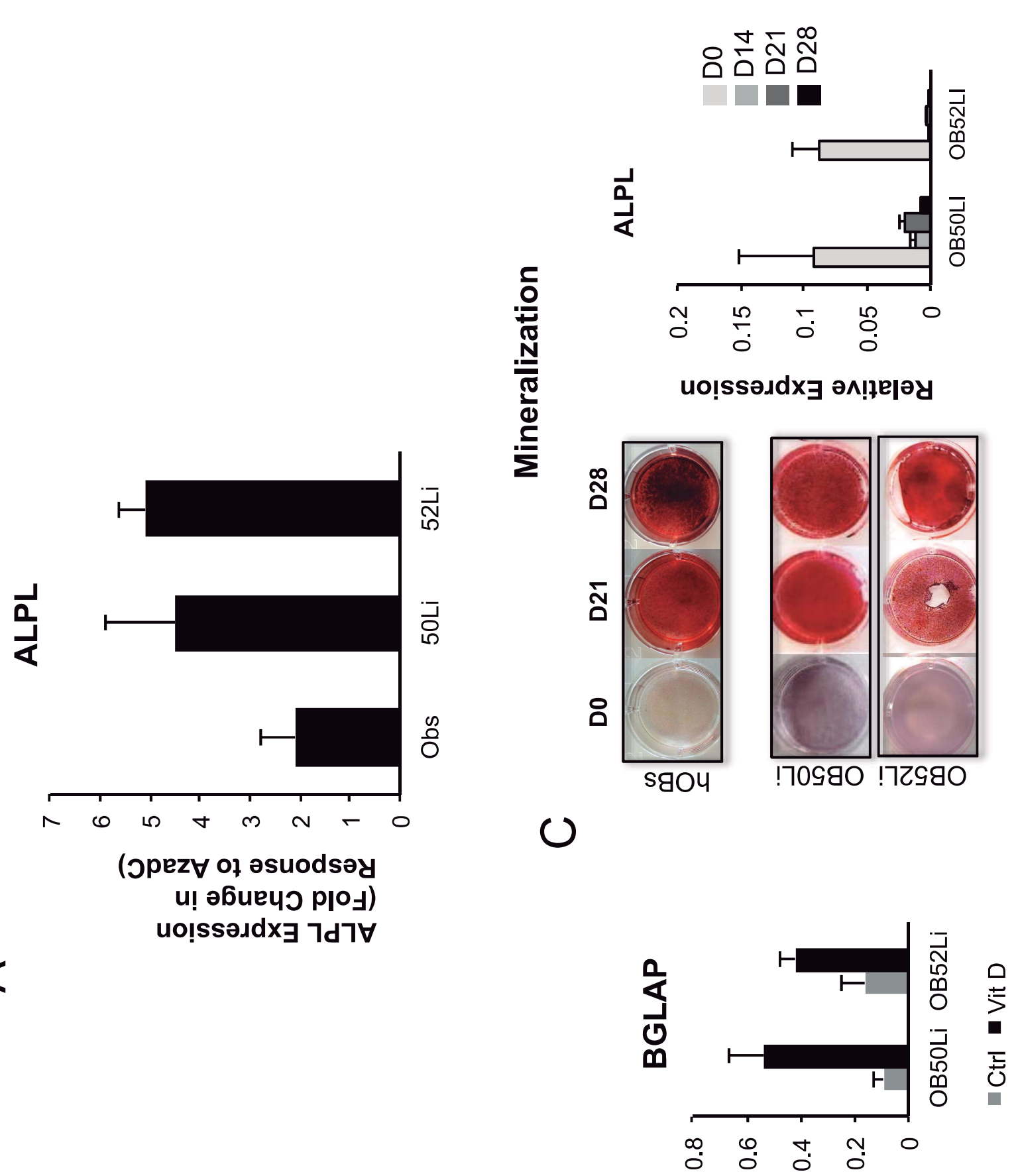

u! әбиечว PIO () uo!ssə.dx 7 רา $\forall$

$\varangle$ uo!ssə.dx 
A

Hematox./Eosin
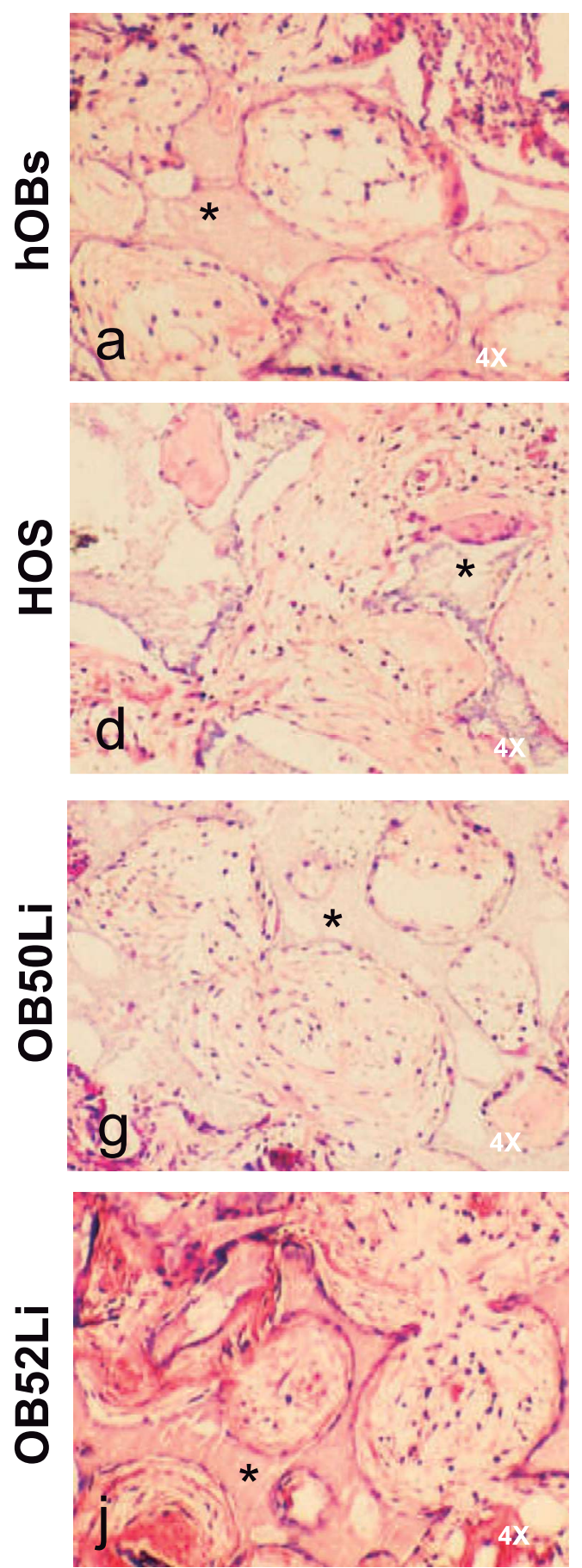

IHQ COL1a1
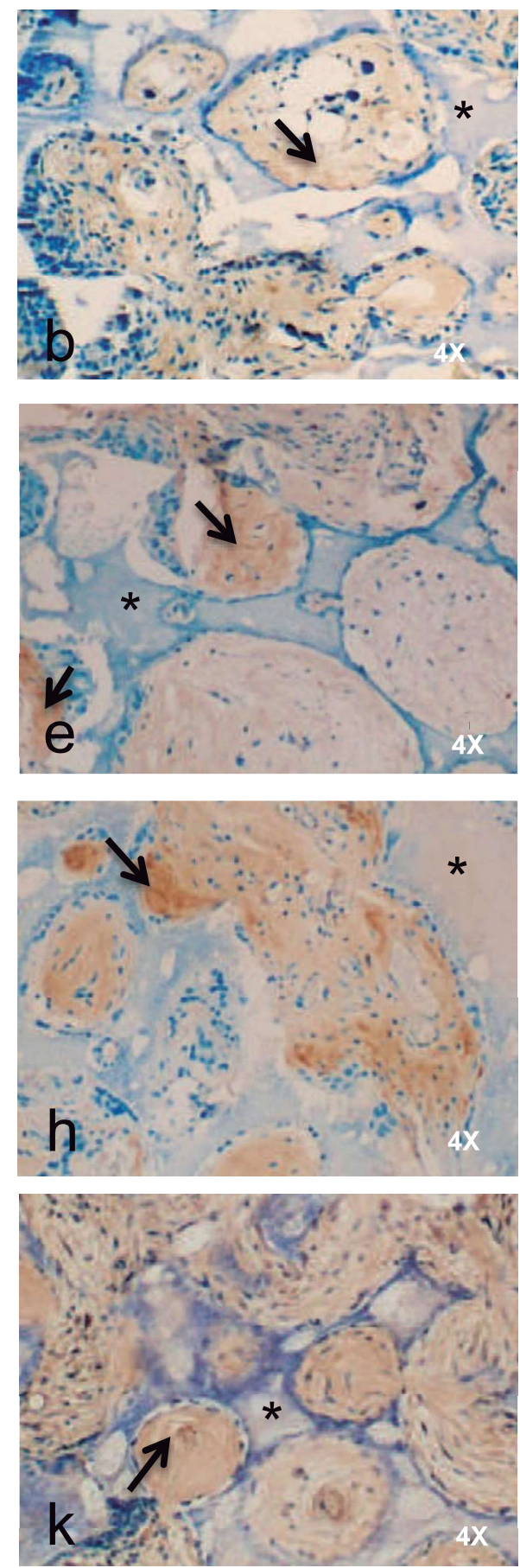

\section{Masson (Bone Matrix)}
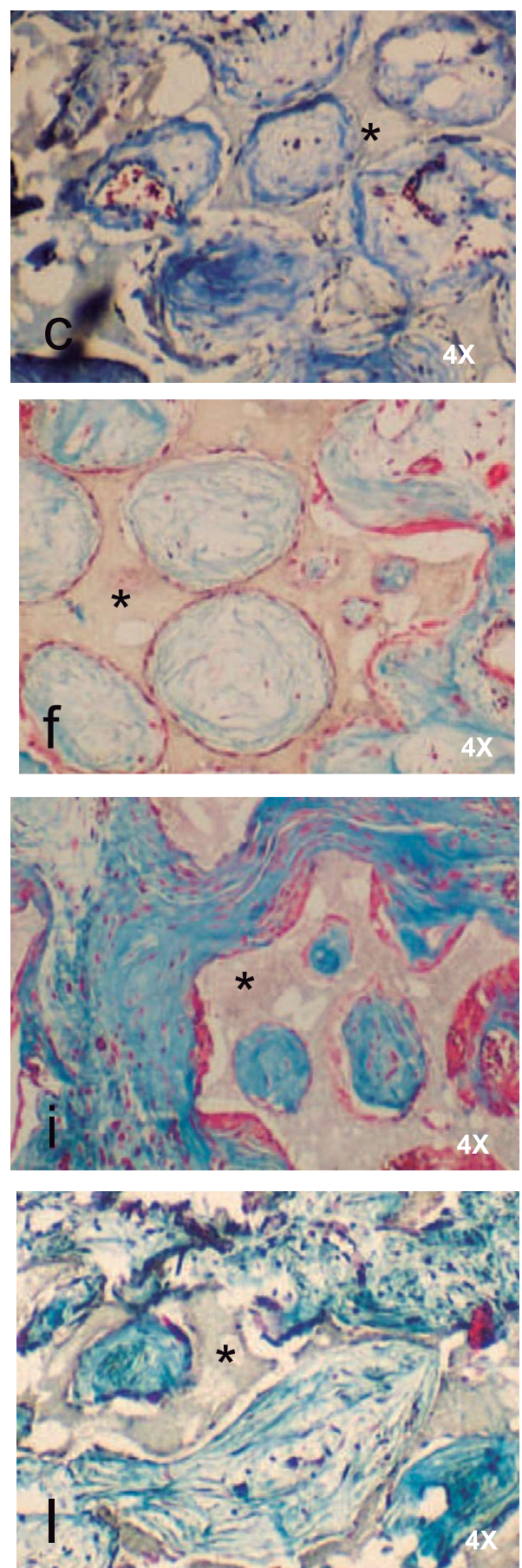

OB50Li

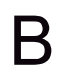

OB52Li

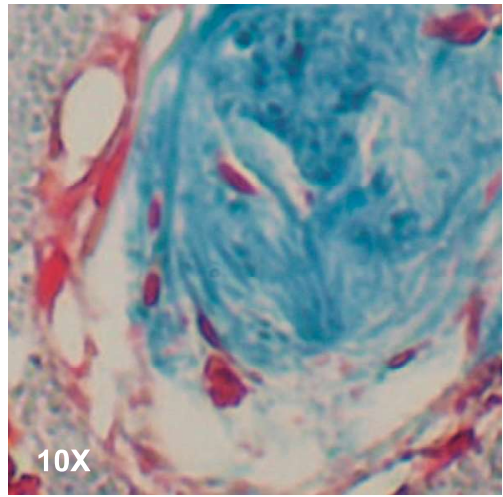

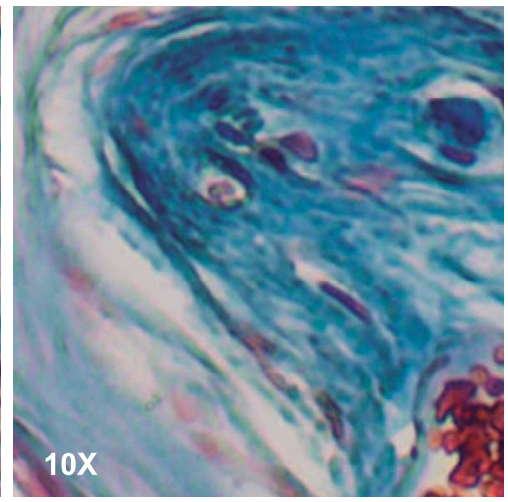


A

Hematox./Eosin

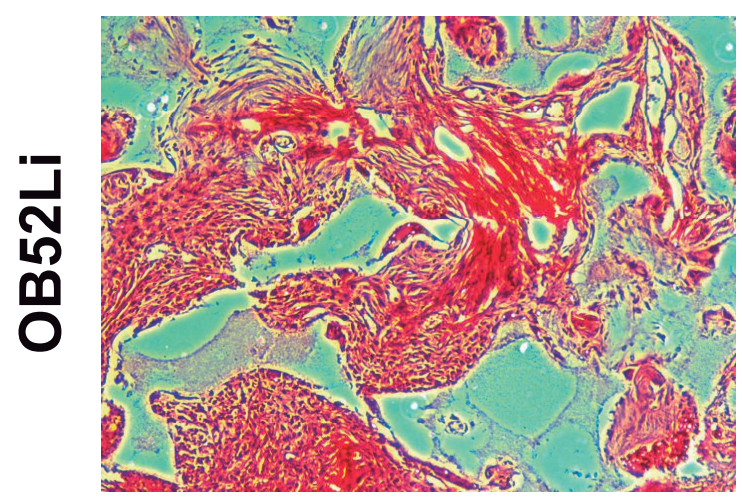

Hematox./Eosin

B

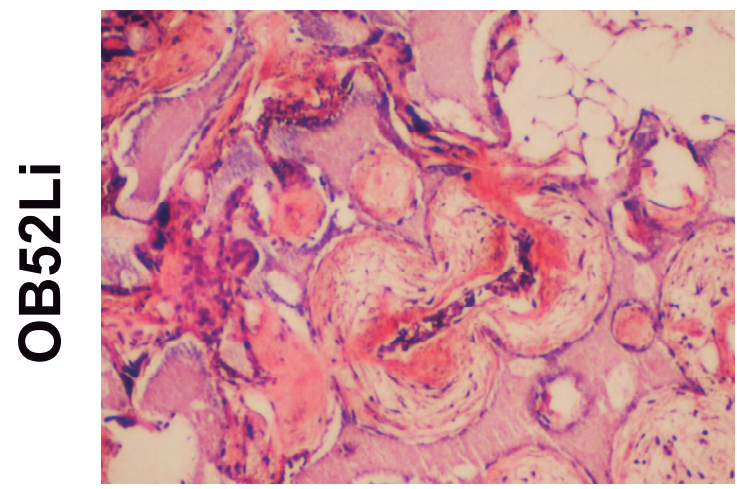

Sirius Red

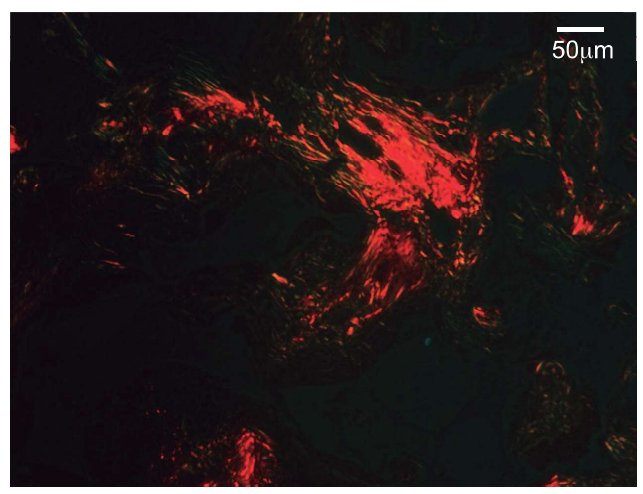

Green Fluorescent light

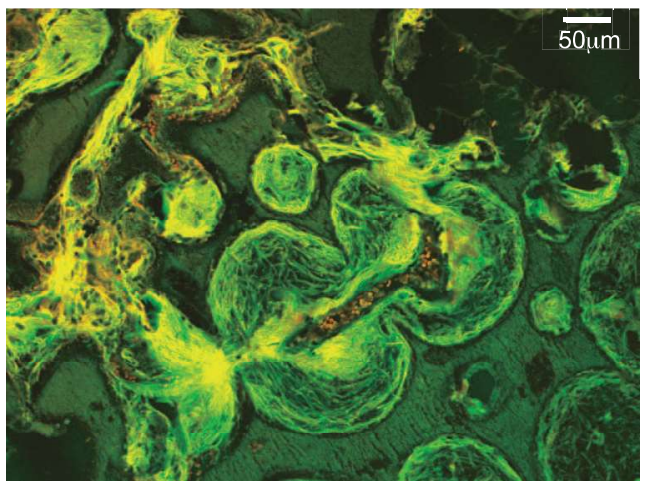

C
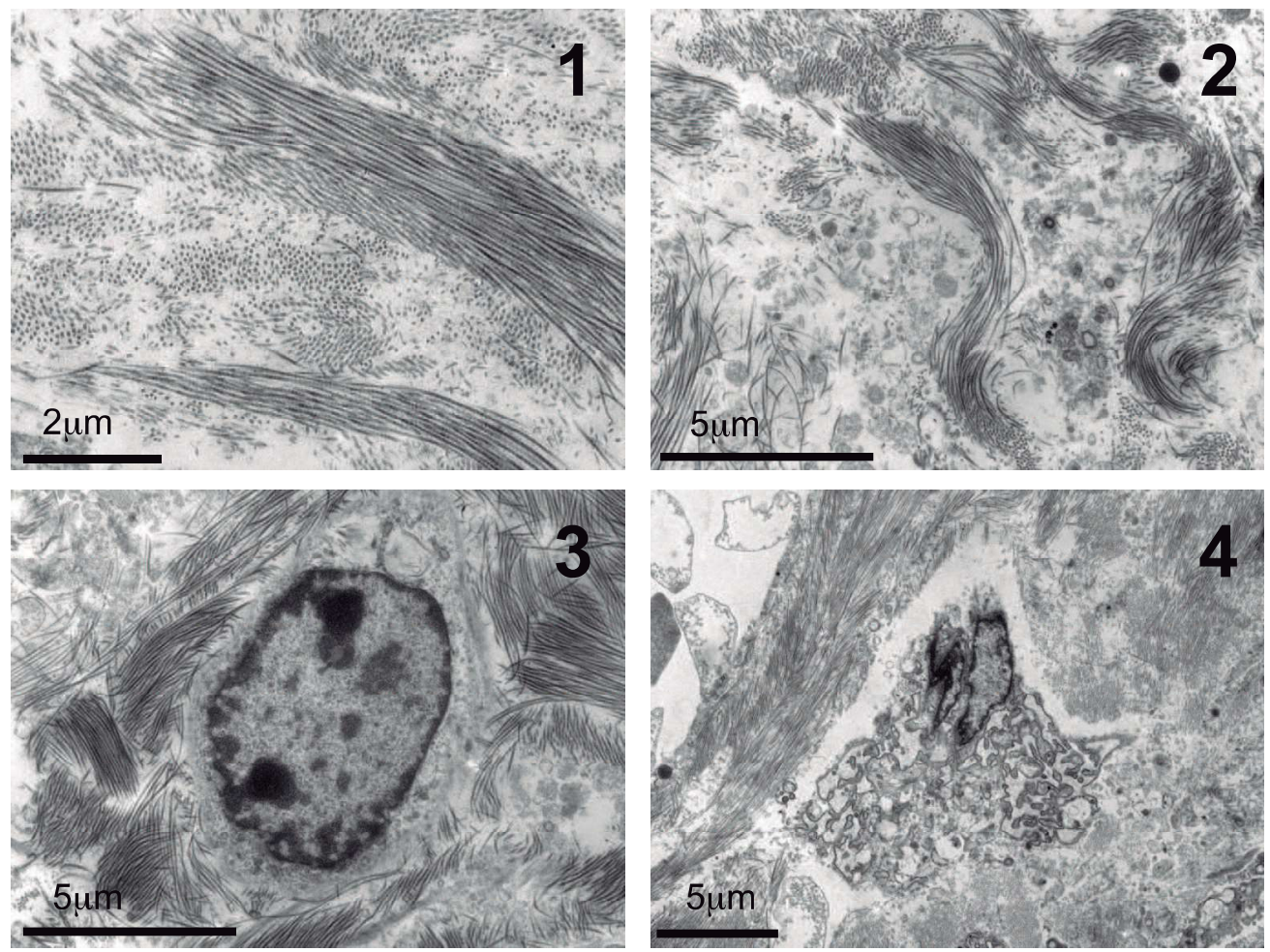

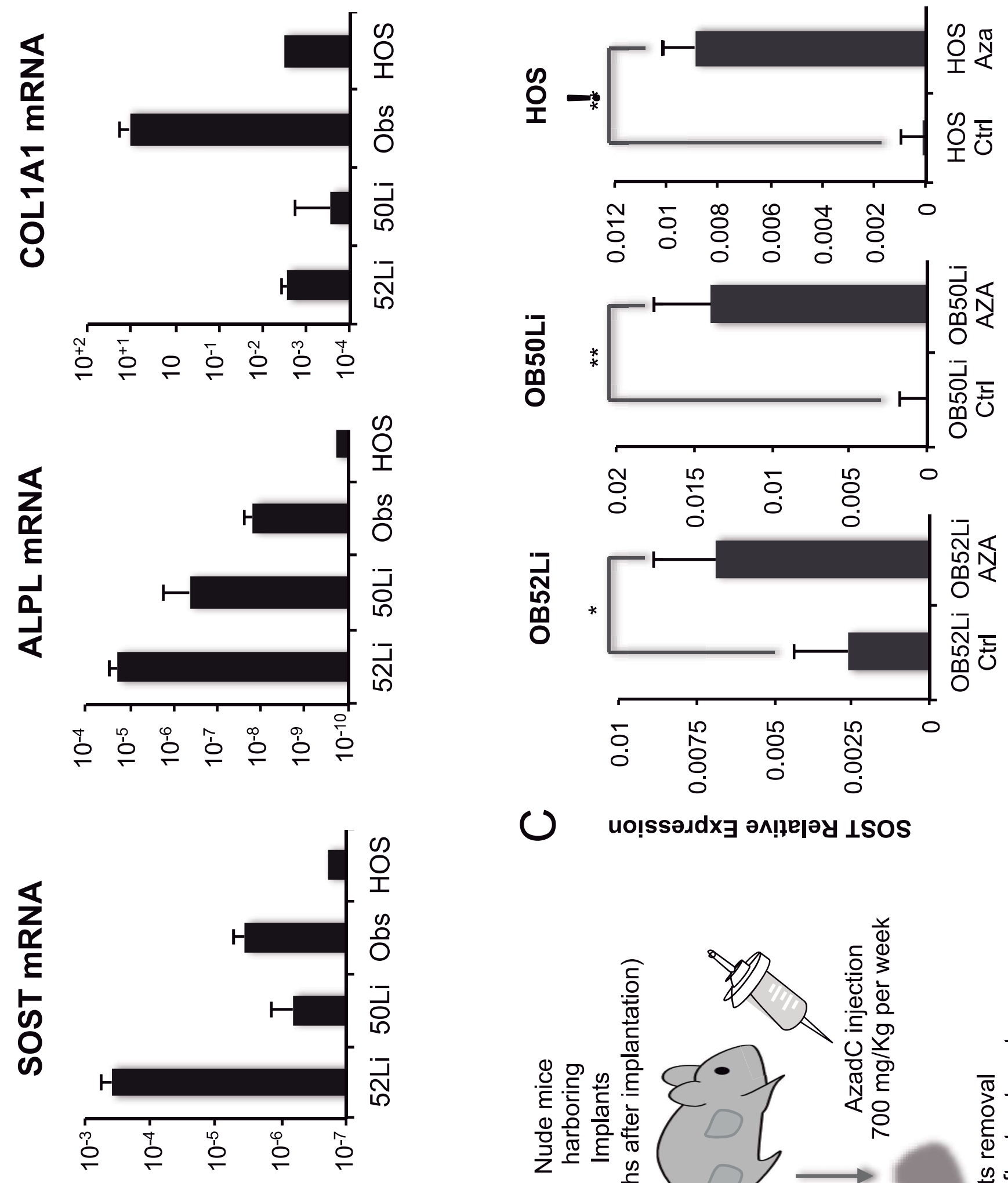

uo!ssə.дdxヨ әм!ฺе|әу

() uо!ssəлdx

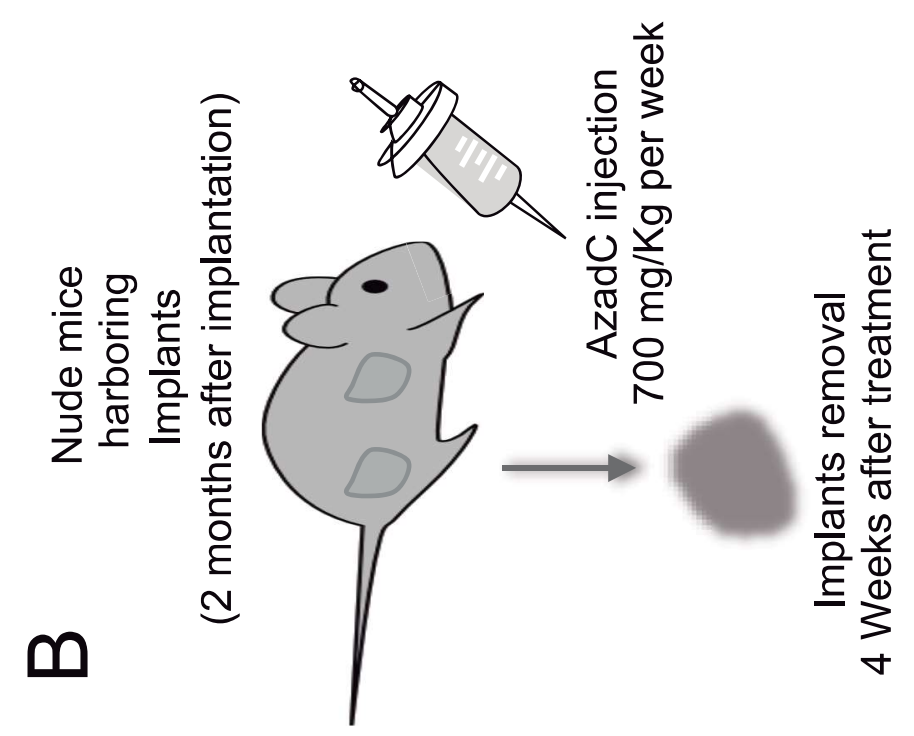

